\title{
Value of Adjuvant Whole-Pelvis Irradiation after Wertheim Hysterectomy for Early-Stage Squamous Carcinoma of the Cervix with Pelvic Nodal Metastasis: A Matched-Control ${ }^{1}$ Study
}

\author{
Walter K. Kinney, M.D., ${ }^{*}, 2$ Ronald D. Alvarez, M.D.,$\dagger$ Gary C. Reid, M.D. ${ }^{3}+$ Mark F. Schray, M.D.,$\S$ \\ Seng-jaw Soong, Ph.D., † George W. Morley, M.D. $\ddagger$ Karl C. Podratz, M.D., Ph.D., ${ }^{*}$ \\ and Hugh M. Shingleton, M.D. $\dagger$ \\ *Department of Obstetrics and Gynecology and \$Division of Radiation Oncology, Mayo Clinic and Mayo Foundation, Rochester, Minnesota \\ 55905; †Department of Obstetrics and Gynecology and the Department of Biostatistics, University of Alabama, Birmingham, Alabama \\ 35294; and $\ddagger$ Department of Obstetrics and Gynecology, University of Michigan, Ann Arbor, Michigan 48109
}

Received November 3, 1988

In a retrospective study, 185 patients with previously untreated stage IB or IIA (International Federation of Gynecology and Obstetrics) squamous cell carcinoma of the cervix were found to have pelvic nodal metastasis at the time of Wertheim hysterectomy and bilateral pelvic lymphadenectomy. Of these patients, 103 received adjuvant whole-pelvis irradiation and 82 received no adjuvant therapy. Median dose of pelvic irradiation was $\mathbf{5 0 0 0}$ cGy. Among the irradiated patients, in $75 \%$ the dose was 5000 cGy or greater. Matching irradiated and nonirradiated patients according to stage, tumor size, and number and location of positive nodes yielded 60 pairs. Mean length of follow-up was 3.9 years for the 60 irradiated patients and 5.8 years for the nonirradiated patients. Kaplan-Meier overall and cancer-specific survival estimates for the two groups were not significantly different $(P>0.30)$. During the follow-up period, 21 surgery-only patients and 22 patients treated with adjuvant radiotherapy had recurrence, but adjuvant radiotherapy decreased the proportion of recurrences occurring in the pelvis alone-27\% compared with $67 \%$ in the surgery-only group $(P=0.01)$. () 1989 Academic Press, Inc.

\section{INTRODUCTION}

During the past four decades, the efficacy of radical hysterectomy and pelvic lymphadenectomy for treating patients with clinical stage IB or IIA cervical carcinoma has been demonstrated repeatedly; however, the pres-

\footnotetext{
1 Presented at the annual meeting of the Society of Gynecologic Oncologists, Maui, Hawaii, February 5-9, 1989.

${ }^{2}$ Current address: Department of Obstetrics and Gynecology, University of California, 1621 Alhambra Boulevard, No. 2500, Sacramento, CA 95816. To whom reprint requests should be addressed.

${ }^{3}$ Current address: 3555 Olentangy River Road, Suite 2080, Columbus, $\mathrm{OH} 43214$.
}

ence of surgically detected pelvic node metastasis has been correlated with moderately high treatment failure rates [1-3]. Because the salvage rate associated with recurrence is poor [4], several adjuvant therapeutic approaches have been proposed in an attempt to decrease recurrences and thus increase patient survival. An example of such therapy has been the sporadic use of postoperative adjuvant pelvic irradiation with the intent of affording these patients a more favorable prognosis.

Using a matched-pairs study design, the authors summarize the pooled experiences from three institutions in an attempt to assess the benefit derived from adjuvant pelvic irradiation after Wertheim hysterectomy for earlystage squamous cell carcinoma of the cervix with pelvic nodal metastasis.

\section{MATERIALS AND METHODS}

The medical records of all patients treated by Wertheim hysterectomy and pelvic lymphadenectomy for clinical stage IB or IIA (International Federation of Gynecology and Obstetrics) carcinoma of the cervix between 1947 and 1986 were reviewed at each institution. Those patients with squamous cell carcinoma metastatic to pelvic lymph nodes were identified and their records were abstracted in detail, examining various surgical and pathologic features, specifics of postoperative pelvic irradiation, times and patterns of recurrence, and overall follow-up status. Follow-up information was obtained by correspondence, telephone, review of death certificates or some combination of these. 
Irradiated and nonirradiated patients were matched according to clinical stage, tumor size, and number and location of positive nodes. Overall and cancer-specific Kaplan-Meier survival estimates [5] were computed for both groups and compared by using the log-rank test. Survival estimates were calculated from the date of operation. Information concerning sites of recurrence was broken down into pelvic, distant, and pelvic-plus-distant sites and compared within the two groups by the $\chi^{2}$ test or Fisher's exact test.

\section{RESULTS}

Pelvic lymph node metastasis was detected at the time of Wertheim hysterectomy in 185 patients with clinical stage IB or IIA squamous cell carcinoma of the cervix; 95 at the Mayo Clinic, 48 at the University of Alabama, and 42 at the University of Michigan. Of these $185 \mathrm{pa}$ tients, 103 received adjuvant pelvic irradiation, and 82 were treated with surgery only. Matching according to clinical stage (stage IB and IIA), tumor size (largest dimension of the cervical lesion $\pm 1 \mathrm{~cm}$ ), and number (1, 2,3 , or $>3$ ) and location of positive nodes (presence or absence of common iliac nodal metastasis) produced 60 pairs (Table 1). Paraaortic nodes were either negative or unknown, with a single exception in the surgery-only group (Table 2).

Distribution according to surgical time intervals for each group is shown in Table 3. The median year of treatment was 1972 for the surgery-only group and 1979 for the group receiving adjuvant irradiation. Radiation dosimetry was available for all matched patients, and patient distribution as a function of the pelvic midplane dose is given in Table 4. The median dose of pelvic irradiation was $5000 \mathrm{cGY}$ (range, $4000-6800 \mathrm{cGy}$ ). Of the irradiated patients, $75 \%$ received $5000 \mathrm{cGy}$ or more. Median age was 42 years (range, 20-73 years) in the surgery-only group and 39 years (23-66 years) in the adjuvant treatment group.

Median duration of follow-up was 5.8 years in the surgery-only group and 3.9 years in the adjuvant irradiation group. Kaplan-Meier survival estimates for overall and cancer-specific survival were not significantly different between the two groups (Figs. 1 and $2 ; P>0.30$ ). Absolute 5-year survival estimates for the two groups were $72 \pm 6 \%$ for surgery only and $64 \pm 7 \%$ for adjuvant irradiation.

During the follow-up period, 21 of the 60 surgery-only patients and 22 of the 60 patients receiving adjuvant irradiation had recurrences. The sites of initial treatment failures are presented in Table 5. The proportion of recurrences occuring in the pelvis only was $67 \%$ in the
TABLE 1

\section{Distribution of Irradiated and Nonirradiated Patients According to Clinical and Pathologic Variables}

\begin{tabular}{|c|c|c|c|c|}
\hline \multirow[b]{3}{*}{ Variable } & \multicolumn{4}{|c|}{ Distribution by type of treatment } \\
\hline & \multicolumn{2}{|c|}{ Surgery only } & \multicolumn{2}{|c|}{$\begin{array}{l}\text { Adjuvant } \\
\text { irradiation }\end{array}$} \\
\hline & Number & $\%$ & Number & $\%$ \\
\hline \multicolumn{5}{|l|}{ Clinical stage } \\
\hline IB & 50 & 83.3 & 50 & 83.3 \\
\hline IIA & 10 & 16.7 & 10 & 16.7 \\
\hline \multicolumn{5}{|c|}{ Size of lesion $(\mathrm{cm})$} \\
\hline$\leqslant 1.0$ & 5 & 8.3 & 8 & 13.3 \\
\hline $1.1-2.0$ & 7 & 11.7 & 8 & 13.3 \\
\hline $2.1-4.0$ & 27 & 45.0 & 26 & 43.3 \\
\hline $4.1-6.0$ & 15 & 25.0 & 10 & 16.7 \\
\hline$>6.0$ & 2 & 3.3 & 6 & 10.0 \\
\hline Unknown & 4 & 6.7 & 2 & 3.3 \\
\hline \multicolumn{5}{|c|}{ Site of positive nodes } \\
\hline Right side & 17 & 28.3 & 22 & 36.7 \\
\hline Left side & 30 & 50.0 & 22 & 36.7 \\
\hline Both sides & 11 & 18.3 & 14 & 23.3 \\
\hline Unknown & 2 & 3.3 & 2 & 3.3 \\
\hline \multicolumn{5}{|c|}{ Positive common iliac nodes } \\
\hline Yes & 4 & 6.7 & 4 & 6.7 \\
\hline No & 19 & 31.7 & 32 & 53.3 \\
\hline Unknown & 37 & 61.7 & 24 & 40.0 \\
\hline \multicolumn{5}{|c|}{ Total number of nodes positive } \\
\hline 1 & 36 & 60.0 & 36 & 60.0 \\
\hline 2 & 14 & 23.3 & 7 & 11.7 \\
\hline 3 & 4 & 6.7 & 11 & 18.3 \\
\hline 4 & 2 & 3.3 & 3 & 5.0 \\
\hline 5 & 2 & 3.3 & 0 & 0.0 \\
\hline 6 & 0 & 0.0 & 1 & 1.7 \\
\hline Unknown & 2 & 3.3 & 2 & 3.3 \\
\hline
\end{tabular}

surgery-only group and $27 \%$ in the adjuvant irradiation group $(P=0.01)$.

Median time to recurrence was 1.85 years for all patients, 1.4 years (range, 0.3-7.0 years) in the surgeryonly group, and 2.1 years (range, $0.4-11.8$ years) in the adjuvant irradiation group. Median time to recurrence

TABLE 2

Distribution of Irradiated and Nonirradiated Patients According to Paraaortic Node Status

\begin{tabular}{lccccc}
\hline & \multicolumn{3}{c}{ Distribution by treatment } \\
\cline { 2 - 3 } & \multicolumn{2}{c}{ Surgery only } & & \multicolumn{2}{c}{ Adjuvant irradiation } \\
\cline { 2 - 3 } \cline { 5 - 6 } Node status & Number & $\%$ & & Number & $\%$ \\
\hline Negative & 31 & 51.6 & & 41 & 68.3 \\
Positive & 1 & 1.7 & & 0 \\
Unknown & 28 & 46.7 & 19 & 31.7 \\
\hline
\end{tabular}


TABLE 3

Distribution of Irradiated and Nonirradiated Patients According to Chronologic Treatment Intervals

\begin{tabular}{lcc}
\hline \multirow{2}{*}{$\begin{array}{l}\text { Time interval } \\
\text { (years) }\end{array}$} & \multicolumn{2}{c}{ Distribution by treatment } \\
\cline { 2 - 3 } Before 1956 & Surgery only & Adjuvant irradiation \\
$1956-1960$ & 4 & 0 \\
$1961-1965$ & 4 & 3 \\
$1966-1970$ & 14 & 1 \\
$1971-1975$ & 7 & 2 \\
$1976-1980$ & 11 & 8 \\
$1981-1986$ & 11 & 26 \\
\hline
\end{tabular}

was 0.9 year for those patients who had recurrence in pelvic and distant sites, 1.5 years for pelvic recurrence alone, and 3.0 years for distant recurrence alone.

\section{DISCUSSION}

The lack of demonstrable survival benefit from adjuvant pelvic irradiation within this population is consistent with previous reports [6-13]. We attempted to eliminate a bias toward irradiating patients with more extensive disease by matching for tumor size and extent of spread.

The overall survival for both groups (5-year survival of 72 and $64 \%$ for surgery only and adjuvant radiation, respectively) is slightly higher than the 50 to $60 \%$ customarily reported for node-positive patients [7-10,12], presumably reflecting the matching process: more patients with a small number of positive nodes were available in the surgery-only group so that a large number $(36 / 60)$ of patients in each group had only one node positive. Previous work has suggested possible benefit from adjuvant irradiation for specific subsets of patients such as those with more than three positive lymph nodes [8]. This study does not contain an adequate number of patients to permit meaningful evaluation of subset survivals. If the increased median follow-up time in the surgery-only group ( 5.8 versus 3.9 years) influenced the

TABLE 4

Distribution of Adjuvant Radiotherapy Dose

\begin{tabular}{|c|c|c|}
\hline \multirow{2}{*}{$\begin{array}{l}\text { Dose } \\
\text { (cGy) }\end{array}$} & \multicolumn{2}{|c|}{ Patients } \\
\hline & Number & $\%$ \\
\hline $4000-4499$ & 4 & 6.7 \\
\hline $4500-4999$ & 11 & 18.3 \\
\hline $5000-5499$ & 22 & 36.7 \\
\hline $5500-5999$ & 9 & 15.0 \\
\hline$>6000$ & 14 & 23.3 \\
\hline
\end{tabular}

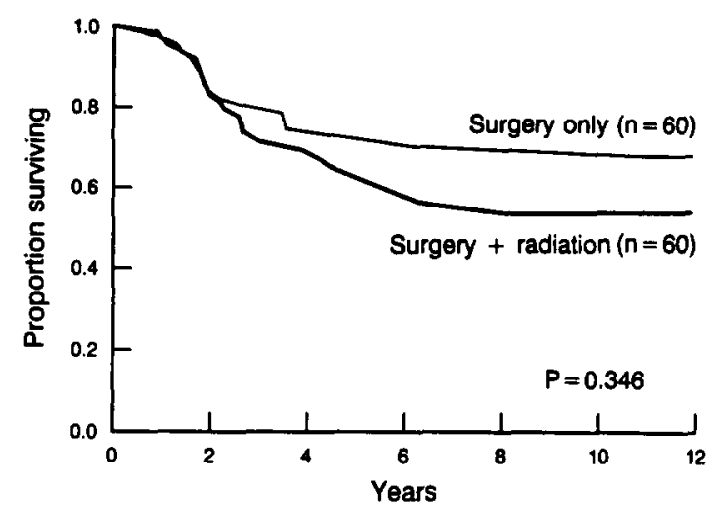

FIG. 1. Overall survival estimates of matched stage IB or IIA cervical carcinoma patients with nodal metastasis, according to treatment.

results, it should have accentuated any diminution in survival in that cohort.

The substantial reduction in pelvic recurrence that we have observed following adjuvant pelvic irradiation is also consistent with previous studies (Table 6). Despite this decrease in pelvic recurrence, long-term survival was not enhanced. The previously unsubstantiated clinical observation that patients who do not have pelvic recurrence fare better in the short term is supported by our observation of a small incrcase in median time to recurrence, from 1.4 to 2.1 years, with the use of adjuvant irradiation. The benefit of decreased pelvic recurrence also is apparent in the doubling of median time to recurrence, from 1.5 years for pelvic failure to 3.0 years in the group with distant recurrence alone.

Statistical power remains a significant concern in this as in previous studies. With a sample size of 60 patients per group, we have a $90 \%$ chance of detecting a $30 \%$ improvement in survival at $P<0.05$ and a $76 \%$ chance of detecting a $25 \%$ improvement in survival at $P<0.05$, assuming a 70\% 5-year survival without treatment.

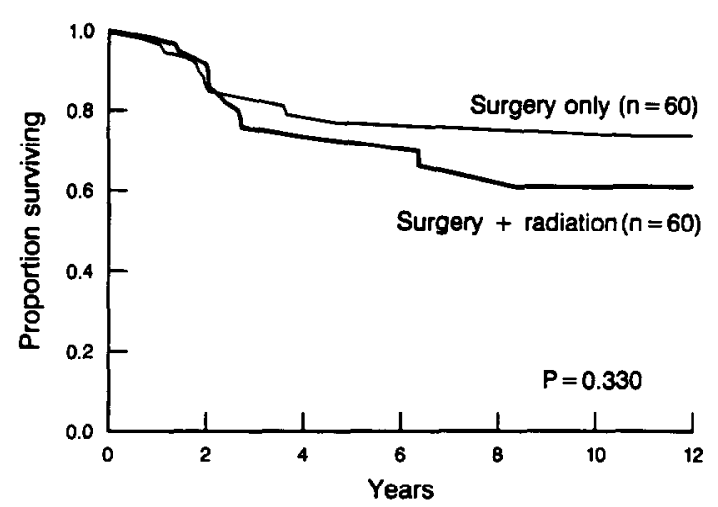

FIG. 2. Cancer-specific survival estimates of matched stage IB or IIA cervical carcinoma patients with nodal metastasis, according to treatment. 
TABLE 5

Distribution of Irradiated and Nonirradiated Patients According to Site of Recurrence

\begin{tabular}{lcccc}
\hline & \multicolumn{3}{c}{ Distribution by treatment } \\
\cline { 2 - 5 } & \multicolumn{2}{c}{ Surgery only } & & \multicolumn{2}{c}{$\begin{array}{c}\text { Adjuvant } \\
\text { irradiation }\end{array}$} \\
\cline { 2 - 6 } Site & Number & $\%$ & Number & $\%$ \\
\hline Pelvis & 14 & 67 & 6 & 27 \\
Distant site & 6 & 29 & 13 & 59 \\
Pelvis + distant site & 1 & 5 & 3 & 14 \\
Any & 21 & 100 & 22 & 100 \\
\hline
\end{tabular}

In summary, we have been able to confirm previous results demonstrating the absence of a discernable survival advantage with adjuvant pelvic irradiation in this setting. According to our results, if such an advantage exists it is probably no larger than $9 \%$ at 5 years $(90 \%$ confidence interval, $-18 \%$ to $+9 \%$ ). Although we also confirmed the decrease in local recurrence after pelvic irradiation and the corresponding increase in short-term survival, the overall 5-year recurrence rates were not different. There is both sound theoretic foundation [15] and preliminary clinical evidence $[16,17]$ to support the contention that small volumes of squamous cell carcinoma of the cervix are potentially chemosensitive. In light of the persistently high recurrence rates found in this study, both in-field and distant, a trial comparison of adjuvant systemic therapy and irradiation in this population appears to be warranted.

\section{REFERENCES}

1. Webb, M. J., and Symmonds, R. E. Wertheim hysterectomy: a rcappraisal, Obstet. Gynecol. 54, 140-145 (1979).

2. Martimbeau, P. W., Kjorstad, K. E., and Iversen, T. Stage IB carcinoma of the cervix, The Norwegian Radium Hospital. II.
Results when pelvic nodes are involved, Obstet. Gynecol. 60, 215218 (1982).

3. Bleker, O. P., Ketting, B. W., van Wayjen-Eecen, B., and Kloosterman, G. J. The significance of microscopic involvement of the parametrium and/or pelvic lymph nodes in cervical cancer stages IB and IIA, Gynecol. Oncol. 16, 56-62 (1983).

4. Webb, M. J., and Symmonds, R. E. Site of recurrence of cervical cancer after radical hysterectomy, Amer. J. Obstet. Gynecol. 138, 813-817 (1980)

5. Kaplan, E. L., and Meier, P. Nonparametric cstimation from incomplete observations, Amer. Stat. Assoc. J. 53, 457-481 (1958).

6. Kelso, J. W., and Funnell, J. D. Combined surgical and radiation treatment of invasive carcinoma of the cervix, Amer. J. Obstet. Gynecol. 116, 205-213 (1973).

7. Rampone, J. F., Klem, V., and Kolstad, P. Combined treatment of stage IB carcinoma of the cervix, Obstet. Gynecol. 41, 163-167 (1973).

8. Masubuchi, K., Tenjin, Y., Kubo, H., and Kimura, M. Five-year cure rate for carcinoma of the cervix uteri: With special reference to the comparison of surgical and radiation therapy, Amer. $J$. Obstet. Gynecol. 103, 566-573 (1969).

9. Piver, M. S., and Chung, W. S. Prognostic significance of cervical lesion size and pelvic node metastases in cervical carcinoma, $\mathrm{Ob}$ stet. Gynecol. 46, 507-510 (1975).

10. Hsu, C-T., Cheng, Y-S., and Su, S-C. Prognosis of uterine cervical cancer with extensive lymph node metastascs: Special emphasis on the value of pelvic lymphadenectomy in the surgical treatment of uterine cervical cancer, Amer. J. Obstet. Gynecol. 114, 954962 (1972).

11. Burch, J. C., and Chalfant, R. L. Preoperative radium irradiation and radical hysterectomy in the treatment of cancer of the cervix, Amer. J. Obstet. Gynecol. 106, 1054-1064 (1970).

12. Morrow, C. P. (Moderator). Is pelvic radiation beneficial in the postoperative management of stage Ib squamous cell carcinoma of the cervix with pelvic node metastasis treated by radical hysterectomy and pelvic lymphadenectomy? A report from the Presidential Panel at the 1979 Annual Meeting of the Society of Gynecologic Oncologists, Gynecol. Oncol. 10, 105-110 (1980).

13. Hogan, W. M., Littman, P., Griner, L., Miller, C. L., and Mikuta, J. J. Results of radiation therapy given after radical hysterectomy, Cancer (Philadelphia) 49, 1278-1285 (1982).

14. Larson, D. M., Stringer, C. A., Copeland, L. J., Gershenson,

TABLE 6

Assessment of Pelvic Recurrences According to Treatment

\begin{tabular}{|c|c|c|c|}
\hline Feature & Hogan et al. [13] & Larson et al. [14] & Morrow [12] \\
\hline \multicolumn{4}{|l|}{ Recurrences } \\
\hline \multicolumn{4}{|l|}{ After adjuvant irradiation } \\
\hline Pelvic & 5 & 0 & 9 \\
\hline All & 10 & 5 & 18 \\
\hline Pelvic & 15 & 2 & 48 \\
\hline All & 16 & 5 & 57 \\
\hline$\%$ Pelvic & 94 & 40 & 84 \\
\hline$P$ for difference between treatments & $<0.001$ & 0.11 & 0.003 \\
\hline \% Decrease in pclvic failure with irradiation & 44 & 40 & 34 \\
\hline
\end{tabular}


D. M., Malone, J. M., Jr., and Rutledge, F. N. Stage IB cervical carcinoma treated with radical hysterectomy and pelvic lymphadenectomy: Role of adjuvant radiotherapy, Obstet. Gynecol. 69, 378-381 (1987).

15. Goldie, J. H., and Coldman, A. J. Quantitative model for multiple levels of drug resistance in clinical tumors, Cancer Treat. Rep. 67, 923-931 (1983).

16. Kim, D. S., Moon, H., and Kang, K. J. Primary chemotherapy and postoperative adjuvant chemotherapy in the treatment of squamous cell carcinoma of the uterine cervix, Gynecol. Oncol. 22, 59-64 (1985).

17. Wertheim, M. S., Hakes, T. B., Daghestani, A. N., Nori, D., Smith, D. H., and Lewis, J. L., Jr. A pilot study of adjuvant therapy in patients with cervical cancer at high risk of recurrence after radical hysterectomy and pelvic lymphadenectomy, J. Clin. Oncol. 3, 912-916 (1985). 